\title{
O.S.P.
}

L'orientation scolaire et professionnelle

$30 / 4 \mid 2001$

Varia

\section{J.-L., Beauvois, N., Dubois, \& W., Doise. La construction sociale de la personne.}

Grenoble : Presses universitaires.

Dahmane Dairi

\section{OpenEdition}

Journals

Electronic version

URL: https://journals.openedition.org/osp/4957

DOI: $10.4000 /$ osp.4957

ISSN: 2104-3795

\section{Publisher}

Institut national d'étude du travail et d'orientation professionnelle (INETOP)

Electronic reference

Dahmane Dairi, "J.-L., Beauvois, N., Dubois, \& W., Doise. La construction sociale de la personne.", L'orientation scolaire et professionnelle [Online], 30/4 | 2001, Online since 31 May 2016, connection on 07 March 2023. URL: http://journals.openedition.org/osp/4957 ; DOI: https://doi.org/10.4000/osp. 4957

This text was automatically generated on 7 March 2023

All rights reserved 


\title{
J.-L., Beauvois, N., Dubois, \& W., Doise. La construction sociale de la personne.
}

Grenoble : Presses universitaires.

\author{
Dahmane Dairi
}

\section{REFERENCES}

Grenoble : Presses universitaires.

1 Quatrième volume d'un traité présentant la recherche empirique et les théories constituant la psychologie sociale, les auteurs ont demandé à des chercheurs français et étrangers d'exposer les réflexions de la psychologie sociale dans trois domaines : la moralité et les valeurs, les constructions identitaires et enfin, les phénomènes de socialisation, d'internalisation et de rationalisation.

2 Est-ce la nature de l'homme qui fait la société ou la société qui fait l'homme? D'emblée, les auteurs, reprenant Stoezel (1963) indiquent que la réalité psychologique individuelle (soi, personnalité...) est déterminée par l'organisation sociale et par les places et rôles qu'elle donne aux gens. Une orientation davantage sociale que psychologique marquait dès le début les comportementalistes (Allport, 1924). Cependant, la société ne se réduit pas à l'entourage, à autrui. Très influent en psychologie sociale, le courant culturaliste se demande comment une société façonne la réalité psychologique de ses membres par la culture.

Pour commencer, les auteurs présentent les différentes théories concernant la morale laquelle se compose d'un système de règles. S'intéressant au « développement moral » chez l'enfant, Piaget énonce que la coopération avec les pairs permet au jeune enfant de passer d'une morale hétéronome à une morale autonome.

4 Kolhberg prolonge l'ceuvre de Piaget et s'en distingue par une description précise de 6 stades du développement moral. Cependant, Gilligan en 1980, a proposé une alternative 
au développement moral kohlbergien dans le cadre du courant post-moderne (Day, \& Tappan, 1996) lequel s'en distingue par une démarche plus hermeneutique qu'empiriste. Depuis 1990, le modèle de Kohlberg a été critiqué à un niveau intraculturel (décrit-il adéquatement la morale occidentale ?) et à un niveau interculturel parce qu'il serait plutôt ethnocentrique (occidental) qu'universel. En fait, ce qui manque souvent à ces théories, c'est une approche psychosociale parce que dans chaque société coexistent plusieurs morales pour lesquelles le critère déterminant n'est pas la culture mais les appartenances sociales des sujets.

Certains travaux soulignent que les individus adhèrent largement aux droits de l'homme et qu'ils acceptent pourtant une limitation des libertés individuelles. Pour Rokeach (1973), il s'agit de conflits de valeurs ; pour Schwartz (1992), il existe avant tout une stabilité de la signification des valeurs au travers des cultures. Bien qu'une recherche effectuée dans 54 pays ait confirmé le modèle de Schwartz, les auteurs soulignent l'absence de la dimension psychosociale dans ces théories (lesquelles devraient être enrichies par les expériences actuellement en cours concernant le passage de sociétés communistes à des sociétés à économie de marché).

Les auteurs se demandent ensuite comment les gens évaluent les décisions prises à leur égard comme étant justes ou injustes. La justice sociale s'intéresse aux résultats obtenus (justice distributive) mais aussi aux procédures utilisées pour allouer les résultats (justice procédurale). La théorie de l'équité d'Adams (1965) s'enracinant dans la théorie de la dissonance cognitive (Festinger, 1957) postule qu'une situation est équitable lorsque ce que nous recevons, nos rétributions, est en lien étroit avec nos contributions. La notion de justice est aujourd'hui un objet central des sciences sociales et constitue un enjeu important dans le maintien et la transformation des liens qui unissent ou opposent les acteurs sociaux. Ceux-ci disposant de différents schèmes de raisonnement pour la distribution et l'échange de biens : l'équité et la solidarité fondent leurs décisions en matière de justice distributive. Le sentiment d'injustice, quant à lui s'élabore à travers une évaluation relative de la situation; c'est pourquoi, l'injustice est plus supportable quand on peut l'expliquer ou la justifier.

7 Dans la deuxième partie, les auteurs rappellent que les travaux de Shérif, Tajfel puis de Turner donnèrent un éclairage nouveau au rôle joué par l'appartenance à un groupe et aux relations intergroupes dans l'élaboration de l'identité sociale des individus. Cependant, ils n'ont pas accordé suffisamment d'intérêt aux rapports asymétriques entre groupes, le statut social apparaissant comme un modérateur de l'effet d'homogéneïté du hors-groupe. La construction de l'identité a aussi un aspect culturel, l'étude du fonctionnement psychologique des individus implique de comprendre les phénomènes de transmission culturelle et dans une perspective interculturelle, de tenir compte de ce qui est commun à toutes les cultures (éthique) et de ce qui est spécifique à une culture (émique). Aujourd'hui, en Occident, la gestion des rapports entre groupes culturels distincts interpelle la psychologie sociale. En pratique, on oppose 2 options : l'assimilation associée au « Melting-Pot » et le multiculturalisme. Ce clivage, réducteur pour les auteurs, Berry tente de le dépasser en proposant un modèle d'acculturation qui s'intéresse aux changements psychologiques et culturels qui se produisent chez les individus au contact de personnes d'une autre culture.

8 Enfin, les auteurs s'interrogent sur le concept d'identité personnelle. Selon eux, le regain d'intérêt pour l'identité est à situer dans l'évolution de l'opposition entre Nature et Culture. Depuis 1970, considérant l'individu comme un acteur, l'identité humaine est 
étudiée comme une construction intéractive et peut être envisagée selon 4 approches théoriques : développementale (Wallon), anthropologique (Erikson), sociocognitive (Tajfel) et en référence au sentiment d'identité de la personne (Malrieu). L'identité personnelle peut être aussi étudiée comme une représentation sociale même si les études allant dans ce sens sont marginales.

Dans la troisième partie, les auteurs développent le concept d'internalisation, forme particulière de la socialisation. Il rassemble les processus cognitifs aboutissant au fait que des comportements ou des abstinences désignés comme utiles par l'environnement social deviennent le fait de la volonté ou d'une disposition interne de la personne. Pour comprendre comment un enfant ou un adulte en vient à se comporter spontanément comme on attend qu'il se comporte, plusieurs théories nous sont proposées. Parmi elles, la psychologie de l'engagement (Kiesler, 1971) énonce que nous pouvons influencer autrui sans recourir à l'autorité ou à la persuasion et sert d'assise au paradigme de la "soumission librement consentie »: les actes précèdent les idées. Ensuite, les auteurs évoquent le thème de la valeur et de l'évaluation en psychologie. Soit l'évaluation est traitée comme relevant de l'inné, soit elle apparaît comme un composant sémantique des significations communiquées par la langue. Cependant, le modèle relativiste complétant le différenciateur sémantique d'Osgood, nous rappelle que la signification évaluative des termes se modifie en fonction du contexte. Bien qu'ayant un aspect descriptif, la personnologie fait également appel à l'évaluation parce qu'elle parle de ce que sont supposés être les gens et de la valeur qu'on leur attribue : valeur dont la signification théorique peut être saisie grâce aux T.I.P. (théories implicites de personnalité). Enfin, pour aborder les concepts de soi et malgré la coexistence de 2 conceptions (cognitiviste et constructiotiviste), le débat sur le statut de l'individu dans le rapport à soi reste posé : est-ce un sujet qui traite de l'information et fait des inférences à partir des comportements ou est-ce un sujet qui construit du sens dans l'intéraction sociale ? Quoiqu'il en soit, les significations découlant des rapports sociaux sont ignorées.

En conclusion, les auteurs nous livrent leur définition de la psychologie sociale : « elle traque ce que les jugements, les affects mais aussi la performance, le comportement dénotent des positions sociales et des appartenances sociales ». 